\title{
ASYMPTOTIC ANALYSIS \\ OF TORSIONAL AND STRETCHING MODES OF THIN RODS
}

\author{
BY \\ H. IRAGO (Departamento de Matemática Aplicada, Universidad de Santiago de Compostela, \\ Spain), \\ N. KERDID (Laboratoire d'Analyse Numérique, Université Pierre et Marie Curie, 75252 Paris \\ Cedex, France), \\ AND \\ J. M. VIAÑO (Departamento de Matemática Aplicada, Universidad de Santiago de Compostela, \\ Spain)
}

\begin{abstract}
In this article, we show that a class of high frequencies of the threedimensional linearized elasticity system in a thin rod and their associated eigenfunctions converge in a precise sense, as the area of the cross section of the rod goes to zero. The limit model is a coupled one-dimensional problem giving the classical equations for torsion and stretching modes in rods.
\end{abstract}

1. Introduction. It is the purpose of this article to show that the standard spectral problem associated to torsional and stretching modes in linear elastic thin rods can be derived mathematically from the standard three-dimensional eigenvalue problem of linear elasticity through a rigourous convergence analysis as the area of the cross section of the rod converges to zero. This result is obtained using a nonstandard asymptotic technique.

The asymptotic method was successfully used to study a large variety of problems in elastic rods. We refer to Trabucho-Viaño [28] for a survey and also Trabucho-Viaño [25]-[27], Álvarez-Dios-Viaño [2], Aganovic-Tutek [1], Bermúdez-Viaño [4], ÁlvarezVázquez-Viaño [3], Rodríguez-Seijo-Viaño [22], Gruais [10], Le Dret [17]-[19]. The mathematical justification of spectral problems in elasticity, using a rigourous asymptotic technique, was firstly done by Ciarlet-Kesavan [7] who obtain the spectral biharmonic problem for plates as a limit of the scaled three-dimensional elasticity problem as the thickness of the plate tends to zero. Their idea was adapted and used to study different

Received April 20, 1998.

2000 Mathematics Subject Classification. Primary 74K10, 74B05, 74G10, 74H45, 49R50.

Key words and phrases. Asymptotic analysis, spectral problem, elastic rod, high frequency modes, torsional vibration, stretching vibration.

This work was performed while the author N. Kerdid was staying at the Departamento de Matemática Aplicada, University of Santiago de C'mpostela, Spain. 
spectral problems: Le Dret [18] for a junction between two plates, Bourquin-Ciarlet [5] and Lods [20] for a plate inserted in a three-dimensional body, Kerdid [13]-[16] for a rod and junction between two rods. All these works are concerned with convergence of low frequency modes of three-dimensional linear elasticity as the area of the cross section tends to zero. The limit problem obtained for rods is the classical spectral problem associated with flexural vibrations of a rod (see Theorem 1). However, the method cannot be used in order to obtain the limit problem for higher frequency modes. This work provides a first contribution to the study of the asymptotic behaviour of the high frequency modes and their associated eigenfunctions in thin linear elastic structures, namely rods.

In fact, we show that, for suitable families of index $\left(l_{\varepsilon}^{m}\right)_{m \in \mathbb{N}^{*}, \varepsilon>0}$, the eigenvalues $\eta_{l_{\varepsilon}^{m}}(\varepsilon)$ of the three-dimensional elasticity problem of a rod with cross section having area $O\left(\varepsilon^{2}\right)$ converge, as $\varepsilon \rightarrow 0$, toward eigenvalues $\eta_{m}(0)$ of a coupled one-dimensional spectral problem giving the classical equations for torsion and stretching vibrations. The corresponding eigenfunctions $u_{\varepsilon}^{l_{\varepsilon}^{m}}(\varepsilon)$ also converge in a certain sense, at least for a subsequence, and the limit displacement is of the form

$$
u^{m}\left(x_{1}, x_{2}, x_{3}\right)=\left(x_{2} \theta^{m}\left(x_{3}\right),-x_{1} \theta^{m}\left(x_{3}\right), \zeta^{m}\left(x_{3}\right)\right),
$$

where $\theta^{m}$ and $\zeta^{m}$ are the torsion angle and the stretching of modes associated with the eigenvalue $\eta_{m}(0)$.

2. The three-dimensional problem. Let $\omega$ be an open, bounded and connected subset of $\mathbb{R}^{2}$ having area $A(\omega)$. Let $\gamma=\partial \omega$ be its boundary, which we assume sufficiently smooth. In order to consider a possibly multiconnected cross section, we write $\gamma=$ $\gamma_{0} \cup \gamma_{1} \cup \cdots \cup \gamma_{p}$, where $\gamma_{0}$ denotes the exterior part of the boundary and $\gamma_{1}, \ldots, \gamma_{p}$ the boundaries of the bounded component of $\mathbb{R}^{2} \backslash \bar{\omega}$, which we denote by $\omega_{1}, \ldots, \omega_{p}$. The outward unitary normal vector will be denoted by $n=\left(n_{1}, n_{2}\right)$. The coordinate system $O x_{1} x_{2}$ will be supposed to be a principal of inertia system associated with the section $\omega$, which means that

$$
\int_{\omega} x_{1} d x_{1} d x_{2}=\int_{\omega} x_{2} d x_{1} d x_{2}=\int_{\omega} x_{1} x_{2} d x_{1} d x_{2}=0 .
$$

We define the second moments of area of $\omega$ as follows:

$$
I_{\alpha}=\int_{\omega} x_{\alpha}^{2} d x_{1} d x_{2} \quad(\alpha=1,2) .
$$

Given $\varepsilon \in \mathbb{R}, 0<\varepsilon \leq 1$ and $L>0$, we define

$$
\omega^{\varepsilon}=\varepsilon \omega, \quad \gamma^{\varepsilon}=\partial \omega^{\varepsilon}=\varepsilon \gamma,
$$

and we denote by $\Omega^{\varepsilon}=\omega^{\varepsilon} \times(0, L)$ the open set that is assumed to be the reference configuration of the rod. We denote by $x^{\varepsilon}=\left(x_{1}^{\varepsilon}, x_{2}^{\varepsilon}, x_{3}^{\varepsilon}\right)=\left(\varepsilon x_{1}, \varepsilon x_{2}, x_{3}\right)$ an arbitrary point in $\Omega^{\varepsilon}$. The parameter $\varepsilon$ identifies the diameter of the cross section $\omega^{\varepsilon}$, which has area $A\left(\omega^{\varepsilon}\right)=\varepsilon^{2} A(\omega)$. We suppose that the rod $\Omega^{\varepsilon}$ is clamped on both its ends $\Gamma_{0}^{\varepsilon}=\omega^{\varepsilon} \times\{0\}$ and $\Gamma_{L}^{\varepsilon}=\omega^{\varepsilon} \times\{L\}$.

The material that constitutes the rod is assumed to be homogeneous and isotropic with Young's modulus $E$, Poisson's ratio $\nu$, and density $\rho$, all independent of $\varepsilon$. Also 
used are Lamé's coefficients $\lambda$ and $\mu$, related to $E$ and $\nu$ by the formulas:

$$
\lambda=\frac{\nu E}{(1+\nu)(1-2 \nu)}, \quad \mu=\frac{E}{2(1+\nu)} .
$$

From now on, we will employ the summation convention on the repeated index; moreover, the Latin indices take their values in the set $\{1,2,3\}$ and the Greek indices in the set $\{1,2\}$ (if the contrary is not mentioned). Also, the superindex $\varepsilon$ will be dropped as $\varepsilon=1$ and ', " are used to denote derivatives with respect to $x_{3}$ for functions only depending on this variable.

Let $V\left(\Omega^{\varepsilon}\right)$ be the following space of admissible displacements:

$$
V\left(\Omega^{\varepsilon}\right)=\left\{v^{\varepsilon}=\left(v_{i}^{\varepsilon}\right) \in\left[H^{1}\left(\Omega^{\varepsilon}\right)\right]^{3}: v_{i}^{\varepsilon}=0 \text { on } \Gamma_{0}^{\varepsilon} \cup \Gamma_{L}^{\varepsilon}\right\} .
$$

The spectral problem in linear elasticity is posed in the following variational form (cf. Sanchez-Hubert-Sanchez-Palencia [24]):

$$
\begin{aligned}
& \left(\eta^{\varepsilon}, u^{\varepsilon}\right) \in \mathbb{R} \times V\left(\Omega^{\varepsilon}\right), \quad u^{\varepsilon} \neq 0, \\
& \int_{\Omega^{\varepsilon}} \sigma_{i j}^{\varepsilon}\left(u^{\varepsilon}\right) e_{i j}^{\varepsilon}\left(v^{\varepsilon}\right) d x^{\varepsilon}=\eta^{\varepsilon} \int_{\Omega^{\varepsilon}} \rho u_{i}^{\varepsilon} v_{i}^{\varepsilon} d x^{\varepsilon}, \quad \text { for all } v^{\varepsilon} \in V\left(\Omega^{\varepsilon}\right),
\end{aligned}
$$

where $\sigma^{\varepsilon}\left(u^{\varepsilon}\right)=\left(\sigma_{i j}^{\varepsilon}\left(u^{\varepsilon}\right)\right)$ is the stress tensor, related with displacement field $u^{\varepsilon}=\left(u_{i}^{\varepsilon}\right)$ by the generalized Hooke's law

$$
\sigma_{i j}^{\varepsilon}\left(u^{\varepsilon}\right)=\lambda e_{p p}^{\varepsilon}\left(u^{\varepsilon}\right) \delta_{i j}+2 \mu e_{I j}^{\varepsilon}\left(u^{\varepsilon}\right),
$$

and $e^{\varepsilon}\left(u^{\varepsilon}\right)=\left(e_{i j}^{\varepsilon}\left(u^{\varepsilon}\right)\right)$ is the linearized strain tensor

$$
e_{i j}^{\varepsilon}\left(u^{\varepsilon}\right)=\frac{1}{2}\left(\partial_{i}^{\varepsilon} u_{j}^{\varepsilon}+\partial_{j}^{\varepsilon} u_{i}^{\varepsilon}\right) \quad\left(\partial_{i}^{\varepsilon}:=\frac{\partial}{\partial x_{i}^{\varepsilon}}\right) .
$$

It is well known (see Raviart-Thomas [21]) that the ellipticity of the precedent problem, due to Korn's inequality, warrants that the eigenvalues of the problem (3) form a sequence that tends to infinity,

$$
0<\eta_{1}^{\varepsilon} \leq \eta_{2}^{\varepsilon} \leq \cdots \leq \eta_{m}^{\varepsilon} \leq \cdots
$$

and there exists an orthonormal Hilbert's basis $\left\{u^{\varepsilon, m}\right\}_{m \geq 1}$ in $\left[L^{2}\left(\Omega^{\varepsilon}\right)\right]^{3}$ made up of associated eigenfunctions, that is,

$$
\int_{\Omega^{\varepsilon}} \sigma_{i j}^{\varepsilon}\left(u^{\varepsilon, m}\right) e_{i j}^{\varepsilon}\left(v^{\varepsilon}\right) d x^{\varepsilon}=\eta_{M}^{\varepsilon} \int_{\Omega^{\varepsilon}} \rho u_{i}^{\varepsilon, m} v_{i}^{\varepsilon} d x^{\varepsilon}, \quad \forall v^{\varepsilon} \in V\left(\Omega^{\varepsilon}\right)
$$

and

$$
\int_{\Omega^{\varepsilon}} \rho u_{i}^{\varepsilon, m} u_{i}^{\varepsilon, n} d x^{\varepsilon}=\delta_{m n}, \quad \text { for all } m, n \geq 1 .
$$

Now, we introduce the following change of variable:

$$
x=\left(x_{1}, x_{2}, x_{3}\right) \in \Omega \mapsto x^{\varepsilon}=\left(\varepsilon x_{1}, \varepsilon x_{2}, x_{3}\right) \in \Omega^{\varepsilon},
$$

and the scaling of the test functions $v^{\varepsilon} \in V\left(\Omega^{\varepsilon}\right)$ that gives $v(\varepsilon) \in V(\Omega)$ defined in the following manner:

$$
v_{\alpha}(\varepsilon)(x)=\varepsilon v_{\alpha}^{\varepsilon}\left(x^{\varepsilon}\right), \quad v_{3}(\varepsilon)(x)=v_{3}^{\varepsilon}\left(x^{\varepsilon}\right)
$$


Then, from (4)-(7), an elemental computation proves that the scaled eigenfunctions $u^{m}(\varepsilon)$ and the eigenvalues $\eta_{m}(\varepsilon)=\eta_{m}^{\varepsilon}$ verify the following spectral problem:

$$
\begin{aligned}
& \left(\eta_{m}(\varepsilon), u^{m}(\varepsilon)\right) \in \mathbb{R} \times V(\Omega), \quad u^{m}(\varepsilon) \neq 0, \\
& b^{\varepsilon}\left(u^{m}(\varepsilon), v\right)=\eta_{m}(\varepsilon)\left[\int_{\Omega} \rho u_{\alpha}^{m}(\varepsilon) v_{\alpha} d x+\varepsilon^{2} \int_{\Omega} \rho u_{3}^{m}(\varepsilon) v_{3} d x\right], \quad v \in V(\Omega),
\end{aligned}
$$

where $b^{\varepsilon}(\cdot, \cdot)$ is the bilinear form

$$
\begin{aligned}
b^{\varepsilon}(u, v)=\varepsilon^{-2} & \int_{\Omega}\left[\lambda e_{\alpha \alpha}(u) e_{\beta \beta}(v)+2 \mu e_{\alpha \beta}(u) e_{\alpha \beta}(v)\right] d x \\
& +\int_{\Omega}\left[\lambda e_{\alpha \alpha}(u) e_{33}(v)+4 \mu e_{3 \beta}(u) e_{3 \beta}(v)+\lambda e_{33}(u) e_{\alpha \alpha}(v)\right] d x \\
& +\varepsilon^{2} \int_{\Omega}(\lambda+2 \mu) e_{33}(u) e_{33}(v) d x
\end{aligned}
$$

with the normalization condition:

$$
\int_{\Omega} u_{\alpha}^{m}(\varepsilon) u_{\alpha}^{n}(\varepsilon)+\varepsilon^{2} \int_{\Omega} u_{3}^{m}(\varepsilon) u_{3}^{n}(\varepsilon)=\delta_{m n}, \quad \text { for all } m, n \geq 1 .
$$

3. About the low frequency modes. The specific form of the rescaled problem (8) is well adapted to a "limit" analysis when $\varepsilon$ approaches zero. A first and interesting analysis was performed by Kerdid [14] using a combination of techniques introduced by Ciarlet-Kesavan [7] for plates and Bermúdez-Viaño [4], Trabucho-Viaño [26], and Le Dret [18] for rods. In this way, a mathematical justification of the one-dimensional flexural spectral problem of rods is obtained. The precise results of convergence are proved in the next theorem.

ThEOREM 1. For each integer $m \geq 1$, there exists $\eta_{m}(0) \in \mathbb{R}$ and $u^{m}(0) \in V(\Omega)-\{0\}$ such that as $\varepsilon \rightarrow 0$,

$$
\varepsilon^{-2} \eta_{m}(\varepsilon) \rightarrow \eta_{m}(0) \quad \text { in } \mathbb{R}
$$

and

$$
u^{m}(\varepsilon) \rightarrow u^{m}(0) \text { strongly in } V(\Omega),
$$

with

$$
u_{\alpha}^{m}(0)(x)=\zeta_{\alpha}^{m}\left(x_{3}\right), \quad u_{3}^{m}(0)(x)=-x_{\alpha}\left(\zeta_{\alpha}^{m}\right)^{\prime}\left(x_{3}\right),
$$

where $\left(\eta_{m}(0), \zeta_{1}^{m}, \zeta_{2}^{m}\right) \in \mathbb{R} \times\left(\left[H_{0}^{2}(0, L)\right]^{2}-\{(0,0)\}\right)$ is the $m$-th eigensolution of the following coupled flexural spectral problem:

$$
\begin{aligned}
& (\eta, \zeta) \in \mathbb{R} \times\left[H_{0}^{2}(0, L)\right]^{2}, \quad \zeta=\left(\zeta_{1}, \zeta_{2}\right) \neq(0,0), \\
& \int_{0}^{L} E I_{\beta} \zeta_{\beta}^{\prime \prime} \chi_{\beta}^{\prime \prime} d x_{3}=\eta A(\omega) \int_{0}^{L} \zeta_{\beta} \chi_{\beta} d x_{3}, \quad \text { for all }\left(\chi_{1}, \chi_{2}\right) \in\left[H_{0}^{2}(0, L)\right]^{2},
\end{aligned}
$$

normalized by

$$
A(\omega) \int_{0}^{L} \rho \zeta_{\alpha}^{m} \zeta_{\alpha}^{n} d x_{3}=\delta_{m n}
$$


If $\eta_{m}(0)$ is a multiple eigenvalue of problem (11) then the convergence of the eigenfunctions $u^{m}(\varepsilon)$ is satisfied only for a subsequence.

Note that if $\zeta_{\beta} \neq 0$ in (11), then it satisfies the classical spectral problem of $O x_{\beta}$ flexural modes of a thin clamped rod, i.e. (cf. Roseau [23]):

$$
E I_{\beta} \zeta_{\beta}^{(4)}=\eta A(\omega) \rho \zeta_{\beta} \quad \text { in }(0, L), \quad \zeta_{\beta}(0)=\zeta_{\beta}(L)=0, \quad \zeta_{\beta}^{\prime}(0)=\zeta_{\beta}^{\prime}(L)=0 .
$$

4. Towards the high frequency modes. Our primary motivation at the starting of this work was to provide a similar method in order to derive a mathematical justification of the classical longitudinal and torsional vibrations models for a rod, namely the following spectral problems, respectively (cf. Roseau [23]):

$$
\begin{gathered}
-E \zeta^{\prime \prime}=\eta \rho \zeta \quad \text { in }(0, L), \quad \zeta(0)=\zeta(L)=0 . \\
-\frac{\mu J}{I_{1}+I_{2}} \theta^{\prime \prime}=\eta \rho \theta \quad \text { in }(0, L), \quad \theta(0)=\theta(L)=0 .
\end{gathered}
$$

In Eq. (15), the constant $J$ is the torsional rigidity coefficient of $\omega$, defined by

$$
J=I_{1}+I_{2}-\int_{\omega}\left[\left(\partial_{1} w\right)^{2}+\left(\partial_{2} w\right)^{2}\right] d x_{1} d x_{2},
$$

where $w$ is the warping function of $\omega$, defined as the only solution of the following Neumann problem $\left(\partial_{n} w\right.$ denotes the normal derivative):

$$
-\Delta w=0 \quad \text { in } \omega, \quad \partial_{n} w=x_{2} n_{1}-x_{1} n_{2} \quad \text { on } \gamma, \quad \int_{\omega} w d x_{1} d x_{2}=0 .
$$

We will use the torsional function of $\omega$, which we denote by $\psi$ and define as follows. Let

$$
p_{1}=\partial_{1} w-x_{2}, \quad p_{2}=\partial_{2} w+x_{1} .
$$

Then

$$
\partial_{\alpha} p_{\alpha}=0 \quad \text { in } \omega, \quad p_{\alpha} n_{\alpha}=0 \quad \text { on } \gamma,
$$

which implies (cf. Girault-Raviart [9]) that there exists a "stream" function $\psi$ such that $p=\operatorname{rot} \psi$, that is,

$$
p_{1}=\partial_{1} w-x_{2}=\partial_{2} \psi, \quad p_{2}=\partial_{2} w+x_{1}=-\partial_{1} \psi .
$$

Moreover, $\psi$ is constant in each component $\gamma_{k}$ of the boundary of $\omega$ and, if we impose it to be zero on $\gamma_{0}$, then it is given as the unique solution of the following elliptic boundary value problem:

$$
\left\{\begin{array}{l}
-\Delta \psi=-\operatorname{rot}(p)=2 \text { in } \omega \\
\psi=0 \text { on } \gamma_{0} \\
\partial_{r} \psi=0 \text { on } \gamma \\
\int_{\gamma_{k}} \partial_{n} \psi d \gamma=2 A\left(\omega_{k}\right), \quad k=1,2, \ldots, p
\end{array}\right.
$$

where $\partial_{\tau} \psi$ denotes the tangential derivative of $\psi$ and $A\left(\omega_{k}\right)$ the area of the hole $\omega_{k}$, 


\begin{tabular}{l|c|c|c|c|} 
& $\lambda_{h, 1}(\varepsilon)$ & $\lambda_{h, 2}(\varepsilon)$ & $\lambda_{h, 3}(\varepsilon)$ & $\lambda_{h, 4}(\varepsilon)$ \\
\hline$\varepsilon=1$ & 391.0 & 2589.0 & $3397.0(*)$ & 8538.0 \\
\hline$\varepsilon=1 / 2$ & 423.0 & 3089.0 & 11283.0 & $13530.0(*)$ \\
\hline$\varepsilon=1 / 4$ & 429.0 & 3237.0 & 12302.0 & 33124.0 \\
\hline
\end{tabular}

TABLE 1. Discrete scaled eigenvalues of 3D-problem

$k=1,2, \ldots, p$. We remark that problem (19) is equivalent to

$$
\left\{\begin{array}{l}
-\Delta \psi=-\operatorname{rot}(p)=2 \text { in } \omega \\
\psi=0 \text { on } \gamma_{0}, \\
\partial_{n} \psi=-p_{2} n_{1}+p_{1} n_{2} \quad \text { on } \gamma_{k}, k=1,2, \ldots, p .
\end{array}\right.
$$

Denoting by $c_{k}$ the (constant) trace of $\psi$ on the boundary $\gamma_{k}$, the following alternative expressions for the torsional rigidity coefficient are obtained:

$$
J=-\int_{\omega} x_{\alpha} \partial_{\alpha} \psi d x_{1} d x_{2}=2 \int_{\omega} \psi d x_{1} d x_{2}+2 c_{k} A\left(\omega_{k}\right) .
$$

In order to obtain some information about the distribution of the eigenvalues of the three-dimensional problem associated with torsional and stretching vibrations, we refer to Irago-Viaño [12] where problem (3) is solved for $\omega^{\varepsilon}=\left(-\frac{\varepsilon}{2}, \frac{\varepsilon}{2}\right) \times\left(-\frac{\varepsilon}{2}, \frac{\varepsilon}{2}\right), L=10$, $E=10^{5}, \nu=0.3, \rho=1$, using a finite element discretization and the inverse power method. Indeed, letting $\lambda_{h, m}(\varepsilon)=\varepsilon^{-2} \eta_{h, m}(\varepsilon)$ where $\eta_{h, m}(\varepsilon)$ is the $m$-th eigenvalue of the discrete version of problem (3), they obtain the results summarized in Table 1.

The symmetry of this particular case implies that the modes associated to flexural vibrations have multiplicity 2 . Also, since $I_{1}=I_{2}$, all eigenvalues of the limit problem (13) have multiplicity 2, and they can be explicitly computed (see Roseau [23] and Table 2).

If we fix the index $m$ and let $\varepsilon$ tend to zero, the values obtained in Tables 1 and 2 confirm the result of Kerdid [15] (see Theorem 1), i.e.:

$$
\lambda_{h, m}(\varepsilon)=\varepsilon^{-2} \eta_{h, m}(\varepsilon) \rightarrow \eta_{m}(0), \quad \text { as } \varepsilon \rightarrow 0 .
$$

On the other hand, the numerical method also gives us the corresponding eigenfunctions.

\begin{tabular}{|c|c|c|c|}
$\eta_{1}(0)$ & $\eta_{2}(0)$ & $\eta_{3}(0)$ & $\eta_{4}(0)$ \\
\hline 415.0 & 3158.0 & 12132.0 & 33153.0 \\
\hline
\end{tabular}

TABLE 2. Limit eigenvalues

From Irago [11] it can be seen that the eigenfunctions associated to the eigenvalues marked with $\left(^{*}\right)$ are torsional deformations. Then, if we denote by $\lambda_{h}^{\mathrm{T}}(\varepsilon)$ the first scaled torsional eigenvalue corresponding to the $\operatorname{rod} \Omega^{\varepsilon}, 0<\varepsilon \leq 1$, we have

$$
\lambda_{h}^{\mathrm{T}}(1)=\lambda_{h, 3}(1), \quad \lambda_{h}^{\mathrm{T}}\left(\frac{1}{2}\right)=\lambda_{h .4}\left(\frac{1}{2}\right), \ldots .
$$


It seems that, in general, $\lambda_{h}^{\mathrm{T}}(\varepsilon)=\lambda_{h, l_{\varepsilon}}(\varepsilon)$ where $l_{\varepsilon} \in \mathbb{N}^{*}$ and $l_{\varepsilon} \rightarrow+\infty$ as $\varepsilon \rightarrow 0$. Consequently,

$$
\lim _{\varepsilon \rightarrow 0} \lambda_{h}^{\mathrm{T}}(\varepsilon)=+\infty
$$

and then all the torsional eigenvalues are concentrated at infinity. This fact explains why they cannot be obtained by the classical asymptotic techniques introduced in CiarletKesavan [7] and used in Kerdid [14] to obtain the flexural eigenvalues.

Our approach, in order to characterize this kind of eigenvalue, consists of varying $l$ and $\varepsilon$ simultaneously. We construct then some families of index $\left\{l_{\varepsilon}\right\}_{\varepsilon>0}$ that depend on $\varepsilon$. In addition, it is well known that the three-dimensional modes of a rod associated with torsional and stretching vibrations have higher frequency than the flexural modes. Then, we alter the coefficient $\varepsilon^{-2}$ from the expressions of the eigenvalues and we identify some sequences $\left(l_{\varepsilon}\right)$ such that $l_{\varepsilon} \rightarrow+\infty$ and $\eta_{l_{\varepsilon}}(\varepsilon)$ converges as $\varepsilon \rightarrow 0$.

The result we obtain is developed in the next section.

5. Convergence analysis for high frequencies. Let $\mathcal{L}$ be the set of applications:

$$
l: \varepsilon \in(0,1] \mapsto l(\varepsilon)=l_{\varepsilon} \in \mathbb{N}^{*},
$$

such that

$$
\lim _{\varepsilon \rightarrow 0} l_{\varepsilon}=+\infty
$$

and for all $\varepsilon \in(0,1]$,

$$
\eta_{l_{\varepsilon}}(\varepsilon)<K_{l}
$$

where $K_{l}$ is a constant independent of $\varepsilon$. In order to show that the set $\mathcal{L}$ is not empty, we use the following lemma (Kerdid [15] for the proof):

LEMMA 1. There exists an increasing sequence of constants $C_{m}>0, m \geq 1$, independent of $\varepsilon$, such that

$$
\eta_{m}(\varepsilon) \leq C_{m} \varepsilon^{2}
$$

with

$$
C_{m} \rightarrow+\infty \quad \text { as } m \rightarrow+\infty \text {. }
$$

Then, we associate to each integer $m \geq 1$ the family of index $\left\{l_{\varepsilon}^{m}\right\}_{\varepsilon>0}$ defined by:

$$
l_{\varepsilon}^{m}=\max \left\{j \in \mathbb{N}^{*}: \eta_{j}(\varepsilon) \leq C_{m}\right\} .
$$

Obviously, $l^{m} \in \mathcal{L}$ for all $m \geq 1$.

Now, we enunciate the main result of this paper.

Theorem 2. For each integer $m \geq 1$, there exists $l^{m} \in \mathcal{L}$ such that, as $\varepsilon \rightarrow 0$,

$$
\eta_{l_{\varepsilon}^{m}}(\varepsilon) \rightarrow \eta_{m}(0)
$$


where $\eta_{m}(0)$ is an eigenvalue of the following limit spectral problem:

$$
\begin{aligned}
& (\eta, \theta, \zeta) \in \mathbb{R} \times\left[H_{0}^{1}(0, L)\right]^{2}, \quad(\theta, \zeta) \neq(0,0) \\
& \mu J \int_{0}^{L} \theta^{\prime} \chi^{\prime} d x_{3}+E A(\omega) \int_{0}^{L} \zeta^{\prime} \varphi^{\prime} d x_{3} \\
& \quad=\eta\left[\left(I_{1}+I_{2}\right) \int_{0}^{L} \rho \theta \chi d x_{3}+A(\omega) \int_{0}^{L} \rho \zeta \varphi d x_{3}\right] \\
& \quad \text { for all }(\chi, \varphi) \in\left[H_{0}^{1}(0, L)\right]^{2} .
\end{aligned}
$$

In addition, there exists an $\varepsilon$-subsequence (still denoted by $\varepsilon$ ) and $u^{m}(0) \in V(\Omega)$ such that, as $\varepsilon \rightarrow 0$,

$$
\begin{gathered}
u_{\alpha}^{l_{\varepsilon}^{m}}(\varepsilon) \rightarrow u_{\alpha}^{m}(0), \quad \text { weakly in } L^{2}\left[0, L ; H^{1}(\omega)\right], \\
\varepsilon u_{3}^{l_{\varepsilon}^{m}}(\varepsilon) \rightarrow u_{3}^{m}(0), \quad \text { weakly in } H^{1}(\Omega) .
\end{gathered}
$$

Moreover, $u^{m}(0)=\left(u_{i}^{m}(0)\right)$ is of the following form:

$$
\begin{aligned}
& u_{1}^{m}(0)\left(x_{1}, x_{2}, x_{3}\right)=x_{2} \theta^{m}\left(x_{3}\right), \\
& u_{2}^{m}(0)\left(x_{1}, x_{2}, x_{3}\right)=-x_{1} \theta^{m}\left(x_{3}\right), \\
& u_{3}^{m}(0)\left(x_{1}, x_{2}, x_{3}\right)=\zeta^{m}\left(x_{3}\right),
\end{aligned}
$$

where $\left(\theta^{m}, \zeta^{m}\right) \in\left[H_{0}^{1}(0, L)\right]^{2}$. If $\left(\theta^{m}, \zeta^{m}\right) \neq(0,0)$, then it is an eigenfunction of problem (23) associated to $\eta_{m}(0)$.

The proof of the previous theorem is decomposed in several steps that we enunciate as lemmas.

Lemma 2. For each $l \in \mathcal{L}$, there exists a constant $C_{l}$ independent of $\varepsilon$, such that for all $\varepsilon, 0<\varepsilon \leq 1$ :

$$
\begin{aligned}
\left\|u_{\alpha}^{l_{\varepsilon}}(\varepsilon)\right\|_{L^{2}\left[0, L ; H^{1}(\omega)\right]} & \leq C_{l}, \\
\left\|\varepsilon u_{3}^{l_{\varepsilon}}(\varepsilon)\right\|_{H^{1}(\Omega)} & \leq C_{l} .
\end{aligned}
$$

Proof. We define the following scaled strain tensor:

$$
\begin{aligned}
\kappa_{\alpha \beta}^{l_{\varepsilon}}(\varepsilon) & =\varepsilon^{-1} e_{\alpha \beta}\left(u^{l_{\varepsilon}}(\varepsilon)\right), \\
\kappa_{\alpha 3}^{l_{\varepsilon}}(\varepsilon) & =e_{\alpha 3}\left(u^{l_{\varepsilon}}(\varepsilon)\right), \\
\kappa_{33}^{l_{\varepsilon}}(\varepsilon) & =\varepsilon e_{33}\left(u^{l_{\varepsilon}}(\varepsilon)\right) .
\end{aligned}
$$

Taking $v=u^{l_{\varepsilon}}(\varepsilon)$ in (8) we have

$$
\begin{aligned}
2 \mu\left\|\kappa^{l_{\varepsilon}}(\varepsilon)\right\|_{\left[L^{2}(\Omega)\right]^{9}}^{2} & \leq b^{\varepsilon}\left(u^{l_{\varepsilon}}(\varepsilon), u^{l_{\varepsilon}}(\varepsilon)\right) \\
& =\rho \eta^{l_{\varepsilon}}(\varepsilon) \leq \rho K_{l} .
\end{aligned}
$$

Therefore,

$$
\left\|\kappa_{i j}^{l_{\varepsilon}}(\varepsilon)\right\|_{L^{2}(\Omega)} \leq C_{l}, \quad 0<\varepsilon \leq 1,
$$


and consequently

$$
\begin{aligned}
\left\|e_{\alpha \beta}\left(u^{l_{\varepsilon}}(\varepsilon)\right)\right\|_{L^{2}(\Omega)} & \leq C_{l} \varepsilon \leq C_{l}, \\
\left\|e_{\alpha 3}\left(u^{l_{\varepsilon}}(\varepsilon)\right)\right\|_{L^{2}(\Omega)} & \leq C_{l}, \\
\left\|e_{33}\left(u^{l_{\varepsilon}}(\varepsilon)\right)\right\|_{L^{2}(\Omega)} & \leq C_{l} \varepsilon^{-1} .
\end{aligned}
$$

Using Korn's inequality in $\left[H^{1}(\omega)\right]^{2}$, we have

$$
\begin{aligned}
\left\|\left(u_{\alpha}^{l_{\varepsilon}}(\varepsilon)\right)\right\|_{L^{2}\left[0, L ;\left[H^{1}(\omega)\right]^{2}\right]}^{2} & =\int_{0}^{L}\left\{\sum_{\alpha, \beta}\left\|\partial_{\alpha} u_{\beta}^{l_{\varepsilon}}(\varepsilon)\right\|_{L^{2}(\omega)}^{2}+\sum_{\alpha}\left\|u_{\alpha}^{l_{\varepsilon}}(\varepsilon)\right\|_{L^{2}(\omega)}^{2}\right\} \\
& \leq c(\omega) \int_{0}^{L}\left\{\sum_{\alpha, \beta}\left\|e_{\alpha \beta}\left(u^{l_{\varepsilon}}(\varepsilon)\right)\right\|_{L^{2}(\omega)}^{2}+\sum_{\alpha}\left\|u_{\alpha}^{l_{\varepsilon}}(\varepsilon)\right\|_{L^{2}(\omega)}^{2}\right\} \\
& =c(\omega)\left\{\sum_{\alpha, \beta}\left\|e_{\alpha \beta}\left(u^{l_{\varepsilon}}(\varepsilon)\right)\right\|_{L^{2}(\Omega)}^{2}+\sum_{\alpha}\left\|u_{\alpha}^{l_{\varepsilon}}(\varepsilon)\right\|_{L^{2}(\Omega)}^{2}\right\} .
\end{aligned}
$$

Now, the first conclusion of (27) is obtained from the first inequality in (29) and from (9). The second inequality of (27) is a direct consequence of Korn's inequality in $V(\Omega)$ for $\bar{u}^{l_{\varepsilon}}(\varepsilon)=\varepsilon u^{l_{\varepsilon}}(\varepsilon)$.

Lemma 3. For each $l \in \mathcal{L}$, there exists an $\varepsilon$-subsequence (still denoted by $\varepsilon$ ), such that, as $\varepsilon \rightarrow 0$ :

$$
\begin{aligned}
\eta_{l_{\varepsilon}}(\varepsilon) \rightarrow \eta(0) & \text { in } \mathbb{R}, \\
u_{\alpha}^{l_{\varepsilon}}(\varepsilon) \rightarrow u_{\alpha}(0) & \text { weakly in } L^{2}\left[0, L ; H^{1}(\omega)\right], \\
\varepsilon u_{3}^{l_{\varepsilon}}(\varepsilon) \rightarrow u_{3}(0) & \text { weakly in } H^{1}(\Omega),
\end{aligned}
$$

and there exist elements $\theta, \zeta_{\alpha}, \zeta \in H_{0}^{1}(0, L)$ such that:

$$
\begin{aligned}
& u_{1}(0)\left(x_{1}, x_{2}, x_{3}\right)=x_{2} \theta\left(x_{3}\right)+\zeta_{1}\left(x_{3}\right), \\
& u_{2}(0)\left(x_{1}, x_{2}, x_{3}\right)=-x_{1} \theta\left(x_{3}\right)+\zeta_{2}\left(x_{3}\right), \\
& u_{3}(0)\left(x_{1}, x_{2}, x_{3}\right)=\zeta\left(x_{3}\right) .
\end{aligned}
$$

Proof. Existence of an $\varepsilon$-subsequence satisfying (30) is directly deduced from Lemma 2. From (29) we obtain

$$
e_{\alpha \beta}\left(u^{l_{\varepsilon}}(\varepsilon)\right) \rightarrow 0, \quad \text { weakly in } L^{2}(\Omega), \text { as } \varepsilon \rightarrow 0 .
$$

Since $u_{\alpha}^{l_{\varepsilon}}(\varepsilon) \rightarrow u_{\alpha}(0)$ weakly in $L^{2}\left[0, L ; H^{1}(\omega)\right]$, it follows that $e_{\alpha \beta}\left(u^{l_{\varepsilon}}(\varepsilon)\right) \rightarrow e_{\alpha \beta}(u(0))$ weakly in $L^{2}(\Omega)$. Then, we have $e_{\alpha \beta}(u(0))=0$, from which (31)-(33) are deduced (cf. Trabucho-Viaño [28]).

Now, we set as in Lemma $2, \bar{u}^{l_{\varepsilon}}(\varepsilon)=\varepsilon u^{l_{\varepsilon}}(\varepsilon)$. From (29) and (30) we deduce that

$$
\bar{u}^{l_{\varepsilon}}(\varepsilon) \rightarrow \bar{u}(0)=\left(0,0, u_{3}(0)\right) \quad \text { weakly in }\left[H^{1}(\Omega)\right]^{3},
$$


and

$$
\begin{aligned}
\left\|e_{\alpha \beta}\left(\bar{u}^{l_{\varepsilon}}(\varepsilon)\right)\right\|_{L^{2}(\Omega)} & \leq C_{l} \varepsilon^{2} \\
\left\|e_{\alpha 3}\left(\bar{u}^{l_{\varepsilon}}(\varepsilon)\right)\right\|_{L^{2}(\Omega)} & \leq C_{l} \varepsilon \\
\left\|e_{33}\left(\bar{u}^{l_{\varepsilon}}(\varepsilon)\right)\right\|_{L^{2}(\Omega)} & \leq C_{l} .
\end{aligned}
$$

Consequently, $e_{\alpha \beta}(\bar{u}(0))=e_{3 \beta}(\bar{u}(0))=0$. Then $u_{3}(0)$ does not depend on the variables $x_{1}$ and $x_{2}$, and (33) is obtained.

Lemma 4. For each $l \in \mathcal{L}$ there exists an $\varepsilon$-subsequence (still denoted by $\varepsilon$ ) such that

$$
\kappa_{i 3}^{l_{\varepsilon}}(\varepsilon) \rightarrow \kappa_{i 3}(0) \quad \text { weakly in } L^{2}(\Omega)
$$

with

$$
\begin{aligned}
& \kappa_{13}(0)\left(x_{1}, x_{2}, x_{3}\right)=-\frac{1}{2} \theta^{\prime}\left(x_{3}\right) \partial_{2} \psi\left(x_{1}, x_{2}\right), \\
& \kappa_{23}(0)\left(x_{1}, x_{2}, x_{3}\right)=\frac{1}{2} \theta^{\prime}\left(x_{3}\right) \partial_{1} \psi\left(x_{1}, x_{2}\right),
\end{aligned}
$$

and

$$
\kappa_{33}(0)\left(x_{1}, x_{2}, x_{3}\right)=\zeta^{\prime}\left(x_{3}\right),
$$

where $\psi$ is the torsional function defined by (19).

Proof. Existence of the $\varepsilon$-subsequence such that (34) holds is a consequence of (29). Equality (37) is obtained from convergence (30) and Eq. (33). In order to prove (35)-(36) we show that $\kappa_{3 \beta}(0) \in L^{2}[0, L ; H(\operatorname{div} ; \omega)]$ and that we have

$$
\begin{array}{ll}
\partial_{\beta} \kappa_{3 \beta}(0)=0 & \text { in } L^{2}\left[0, L ; L^{2}(\omega)\right], \\
\kappa_{3 \beta}(0) n_{\beta}=0 & \text { in } L^{2}\left[0, L ; H^{-\frac{1}{2}}(\gamma)\right] .
\end{array}
$$

In fact, taking $v=\left(0,0, v_{3}\right), v_{3} \in H^{1}(\Omega), v_{3}=0$ in $\Gamma_{0} \cup \Gamma_{L}$ in (8), it gives

$$
\begin{gathered}
\int_{\Omega}\left[\lambda e_{\rho \rho}\left(u^{l_{\varepsilon}}(\varepsilon)\right) \partial_{3} v_{3}+2 \mu e_{3 \beta}\left(u^{l_{\varepsilon}}(\varepsilon)\right) \partial_{\beta} v_{3}\right] d x \\
+\varepsilon^{2} \int_{\Omega}(\lambda+2 \mu) e_{33}\left(u^{l_{\varepsilon}}(\varepsilon)\right) \partial_{3} v_{3} d x \\
=\eta_{l_{\varepsilon}}(\varepsilon) \varepsilon^{2} \int_{\Omega} \rho u_{3}^{l_{\varepsilon}}(\varepsilon) v_{3} d x .
\end{gathered}
$$

Passing to the limit as $\varepsilon \rightarrow 0$ and on account of (29), we obtain

$$
2 \mu \int_{\Omega} \kappa_{3 \beta}(0) \partial_{\beta} v_{3} d x=0
$$

The strong interpretation of (39) gives (38). Then, by using the div-curl theorem, there exists an only function $\tau \in L^{2}\left[0, L ; H^{1}(\omega)\right]$ such that

$$
\kappa_{31}(0)=\partial_{2} \tau, \quad \kappa_{32}(0)=-\partial_{1} \tau \quad \text { in } L^{2}\left[0, L ; L^{2}(\omega)\right],
$$

and for almost every $x_{3} \in[0, L]$, the trace of $\tau\left(x_{3}\right)$ on $\gamma_{0}$ is zero and on $\gamma_{k}$ is a constant $c_{k}\left(x_{3}\right), k=1,2, \ldots, p$. Moreover, a.e. in $[0, L] \tau$ is the only solution of the following 
problem:

$$
\begin{aligned}
\Delta \tau=\operatorname{rot}\left(\kappa_{3 \beta}(0)\right) & \text { in } \omega \\
\tau=0 & \text { on } \gamma_{0}, \\
\partial_{n} \tau=-\kappa_{32}(0) n_{1}+\kappa_{31}(0) n_{2} & \text { on } \gamma_{k}, k=1,2, \ldots, p .
\end{aligned}
$$

From (28) we have

$$
\begin{aligned}
\operatorname{rot}\left(\kappa_{3 \beta}^{l_{\varepsilon}}(\varepsilon)\right) & =\partial_{2} e_{13}\left(u^{l_{\varepsilon}}(\varepsilon)\right)-\partial_{1} e_{23}\left(u^{l_{\varepsilon}}(\varepsilon)\right) \\
& =\frac{1}{2} \partial_{3}\left(\partial_{2} u_{1}^{l_{\varepsilon}}(\varepsilon)-\partial_{1} u_{2}^{l_{\varepsilon}}(\varepsilon)\right) \\
& =\frac{1}{2} \partial_{3} \operatorname{rot}\left(u_{\beta}^{l_{\varepsilon}}(\varepsilon)\right) .
\end{aligned}
$$

Using now (34) and Lemma 3 we have

$$
\operatorname{rot}\left(\kappa_{3 \beta}^{l_{\varepsilon}}(\varepsilon)\right) \rightarrow \operatorname{rot}\left(\kappa_{3 \beta}(0)\right)=\frac{1}{2} \partial_{3} \operatorname{rot}\left(u_{\beta}(0)\right)=\theta^{\prime}, \quad \text { weakly in } H^{-1}(\Omega) .
$$

Substituting the previous expression in (41) and from definition (20) of the torsional function $\psi$, we obtain

$$
\tau\left(x_{3}\right)=-\frac{1}{2} \theta^{\prime}\left(x_{3}\right) \psi, \quad \text { a.e. } x_{3} \in[0, L] .
$$

The result is now concluded from (40).

Lemma 5. For each $l \in \mathcal{L}$ there exists an $\varepsilon$-subsequence (still denoted by $\varepsilon$ ) such that as $\varepsilon \rightarrow 0$

$$
\kappa_{\alpha \beta}^{l_{\varepsilon}}(\varepsilon) \rightarrow \kappa_{\alpha \beta}(0) \quad \text { weakly in } L^{2}(\Omega),
$$

where for almost every $x_{3} \in[0, L],\left(\kappa_{\alpha \beta}(0)\left(x_{3}\right)\right)$ is a solution of the following problem:

$$
\begin{aligned}
& \int_{\omega}\left[\lambda \kappa_{\rho \rho}(0)\left(x_{3}\right) \delta_{\alpha \beta}+2 \mu \kappa_{\alpha \beta}(0)\left(x_{3}\right)\right] e_{\alpha \beta}(\varphi)=-\lambda \zeta^{\prime}\left(x_{3}\right) \int_{\omega} e_{\rho \rho}(\varphi), \\
& \quad \text { for all } \varphi=\left(\varphi_{\alpha}\right) \in\left[H^{1}(\omega)\right]^{2} .
\end{aligned}
$$

Then,

$$
\int_{\omega} \kappa_{\rho \rho}(0)\left(x_{3}\right) d x_{1} d x_{2}=\frac{-\lambda}{\lambda+\mu} A(\omega) \zeta^{\prime}\left(x_{3}\right) .
$$

Proof. Existence of the $\varepsilon$-subsequence $\kappa_{\alpha \beta}^{l_{\varepsilon}}(\varepsilon)$ weakly convergent in $L^{2}(\Omega)$ is obtained from (29). Taking $v=\left(v_{1}, v_{2}, 0\right), v_{\alpha} \in H^{1}(\Omega), v_{\alpha}=0$ on $\Gamma_{0} \cup \Gamma_{L}$ in (8), one has:

$$
\begin{aligned}
& \varepsilon^{-2} \int_{\Omega}\left[\lambda e_{\alpha \alpha}\left(u^{l_{\varepsilon}}(\varepsilon)\right) e_{\beta \beta}(v)+2 \mu e_{\alpha \beta}\left(u^{l_{\varepsilon}}(\varepsilon)\right) e_{\alpha \beta}(v)\right] d x \\
& \quad+\int_{\Omega}\left[2 \mu e_{3 \beta}\left(u^{l_{\varepsilon}}(\varepsilon)\right) \partial_{3} v_{\beta} d x+\lambda e_{33}\left(u^{l_{\varepsilon}}(\varepsilon)\right) e_{\alpha \alpha}(v)\right] d x \\
& =\eta_{l_{\varepsilon}}(\varepsilon) \int_{\Omega} \rho u_{\alpha}^{l_{\varepsilon}}(\varepsilon) v_{\alpha} d x .
\end{aligned}
$$

Multiplying by $\varepsilon$ and passing to the limit as $\varepsilon \rightarrow 0$, we obtain

$$
\begin{aligned}
\int_{\Omega}\left[\lambda \kappa_{\alpha \alpha}(0) e_{\beta \beta}(v)+2 \mu \kappa_{\alpha \beta}(0) e_{\alpha \beta}(v)\right] d x+\int_{\Omega} \lambda \kappa_{33}(0) e_{\alpha \alpha}(v) d x & =0 \\
\text { for all }\left(v_{\alpha}\right) \in\left[H^{1}(\Omega)\right]^{2}, \quad v_{\alpha} & =0 \text { on } \Gamma_{0} \cup \Gamma_{L} .
\end{aligned}
$$


Taking $v_{\alpha}\left(x_{1}, x_{2}, x_{3}\right)=\chi\left(x_{3}\right) \varphi_{\alpha}\left(x_{1}, x_{2}\right), \varphi_{\alpha} \in H^{1}(\omega), \chi \in H_{0}^{1}(0, L)$, we deduce for almost every $x_{3} \in[0, L]$ :

$$
\begin{aligned}
& \int_{\omega}\left[\lambda \kappa_{\rho \rho}(0) \delta_{\alpha \beta}+2 \mu \kappa_{\alpha \beta}(0)\right] e_{\alpha \beta}(\varphi)=-\lambda \int_{\omega} \kappa_{33}(0) \delta_{\alpha \beta} e_{\alpha \beta}(\varphi), \\
& \quad \text { for all } \varphi=\left(\varphi_{\alpha}\right) \in\left[H^{1}(\omega)\right]^{2} .
\end{aligned}
$$

Now, expression (44) is concluded from (37). In order to obtain (45), it suffices to take $\varphi_{\alpha}=x_{\alpha}$ in (44).

Using over-refined arguments, the elements $\kappa_{\alpha \beta}(0)$ obtained in the previous lemma can be completely characterized below.

LEMma 6 . The elements $\kappa_{\alpha \beta}(0)$ are of the following form:

$$
\begin{aligned}
& \kappa_{12}(0)\left(x_{1}, x_{2}, x_{3}\right)=0 \\
& \kappa_{11}(0)\left(x_{1}, x_{2}, x_{3}\right)=\kappa_{22}(0)\left(x_{1}, x_{2}, x_{3}\right)=\frac{-\lambda}{2(\lambda+\mu)} \zeta^{\prime}\left(x_{3}\right) .
\end{aligned}
$$

Proof. It is similar to that of Kerdid [15], Lemma 5.

Lemma 7. For each $l \in \mathcal{L}$, if $\eta(0)$ of Lemma 3 is not zero, then $\zeta_{\alpha}=0$.

Proof. Taking a test function of the form $v=\left(\chi_{1}, \chi_{2}, 0\right), \chi_{\alpha} \in H_{0}^{1}(0, L)$ in (8) and passing to the limit, we obtain

$$
4 \mu \int_{\Omega} \kappa_{\alpha 3}(0) \chi_{\alpha}^{\prime} d x=\eta(0) \int_{\Omega} u_{\alpha}(0) \chi_{\alpha} d x=\eta(0) A(\omega) \int_{0}^{L} \zeta_{\alpha} \chi_{\alpha} d x_{3} .
$$

Using (35)-(36) and the property $\int_{\omega} \partial_{\alpha} \psi=0$, we deduce

$$
\int_{\Omega} k_{\alpha 3}(0) \chi_{\alpha}^{\prime} d x=\frac{(-1)^{\alpha}}{2} \int_{\omega} \partial_{\beta} \psi d x_{1} d x_{2} \int_{0}^{L} \theta^{\prime}\left(x_{3}\right) \chi_{\alpha}^{\prime} d x_{3}=0, \quad \beta \neq \alpha .
$$

Then

$$
\eta(0) A(\omega) \int_{0}^{L} \zeta_{\alpha} \chi_{\alpha} d x_{3}=0, \quad \text { for all } \chi_{\alpha} \in H_{0}^{1}(0, L),
$$

from which we conclude $\zeta_{\alpha}=0$.

Lemma 8. The twist angle $\theta \in H_{0}^{1}(0, L)$ and the stretching displacement $\zeta \in H_{0}^{1}(0, L)$ satisfy

$$
\begin{aligned}
\mu J \int_{0}^{L} \theta^{\prime} \chi^{\prime} d x_{3}+ & E A(\omega) \int_{0}^{L} \zeta^{\prime} \varphi^{\prime} d x_{3} \\
& =\eta(0)\left[\left(I_{1}+I_{2}\right) \int_{0}^{L} \rho \theta \chi d x_{3}+A(\omega) \int_{0}^{L} \rho \zeta \varphi d x_{3}\right] \\
& \text { for all }(\chi, \varphi) \in\left[H_{0}^{1}(0, L)\right]^{2}
\end{aligned}
$$


Proof. Taking a test function $v=\left(x_{2} \chi,-x_{1} \chi, \varepsilon^{-1} \varphi\right), \chi, \varphi \in H_{0}^{1}(0, L)$, in Eq. (8) and passing to the limit, we have

$$
\begin{gathered}
4 \mu \int_{\Omega} \kappa_{\alpha 3}(0) e_{\alpha 3}(v) d x+\int_{\Omega}\left[2 \mu \kappa_{33}(0)+\lambda \kappa_{i i}(0)\right] \varphi^{\prime} d x \\
=\eta(0)\left\{\int_{\Omega} \rho u_{\alpha}(0) v_{\alpha} d x+\int_{\Omega} u_{3}(0) \varphi d x\right\} .
\end{gathered}
$$

By substituting expressions (35)-(37) and (45), we obtain

$$
\begin{aligned}
& -\mu\left(\int_{\omega} x_{\alpha} \partial_{\alpha} \psi d x_{1} d x_{2}\right) \int_{0}^{L} \theta^{\prime} \chi^{\prime} d x_{3}+\left(2 \mu+\lambda-\frac{\lambda^{2}}{\lambda+\mu}\right) A(\omega) \int_{0}^{L} \zeta^{\prime} \varphi^{\prime} d x_{3} \\
& \quad=\eta(0)\left\{\left(I_{1}+I_{2}\right) \int_{0}^{L} \rho \theta \chi d x_{3}+A(\omega) \int_{0}^{L} \rho \zeta \varphi d x_{3}\right\},
\end{aligned}
$$

which coincides with Eq. (48) using (21) and (2).

In order to deduce the final results, we define $\lambda_{m}(\varepsilon)=\varepsilon^{-2} \eta_{m}(\varepsilon)$. Then $\lambda_{m}(\varepsilon)$ are the eigenvalues of the following problem:

$$
\begin{aligned}
b^{\varepsilon}(u(\varepsilon), v)=\lambda(\varepsilon) & \left\{\varepsilon^{2} \int_{\Omega} \rho u_{\alpha}(\varepsilon) v_{\alpha} d x+\varepsilon^{4} \int_{\Omega} \rho u_{3}(\varepsilon) v_{3} d x\right\}, \\
& \text { for all } v \in V(\Omega),
\end{aligned}
$$

and verify

$$
\begin{gathered}
0<\lambda_{1}(\varepsilon) \leq \lambda_{2}(\varepsilon) \leq \cdots \leq \lambda_{m}(\varepsilon) \leq \cdots, \\
\lambda_{m}(\varepsilon) \rightarrow_{\varepsilon \rightarrow 0}+\infty .
\end{gathered}
$$

Continuous dependence of $\lambda_{m}(\varepsilon)$ with respect to $\varepsilon$ in $(0,1]$ is well known. For $\varepsilon=0$, continuity was proved by Kerdid [15] (see Theorem 1 above). In this framework we have the following lemma, the proof of which is adapted from Castro-Zuazua [6].

LEMmA 9. For each $m \geq 1$, there exists $l^{m} \in \mathcal{L}$ and a subsequence $\varepsilon_{j} \rightarrow 0$ such that

$$
\varepsilon^{2} \lambda_{l_{\varepsilon_{j}}^{m}}(\varepsilon)=\eta_{l_{\varepsilon_{j}}^{m}}(\varepsilon)=\eta_{m}(0)
$$

where $\eta_{m}(0)$ is the $m$-th eigenvalue of the coupled limit problem (23).

Proof. Suppose that for any $\varepsilon_{j} \rightarrow 0$ and for any $l^{m} \in \mathcal{L}$,

$$
\eta_{\varepsilon_{j}}\left(\varepsilon_{j}\right)=\varepsilon_{j}^{2} \lambda_{l_{\varepsilon_{j}}^{m}}\left(\varepsilon_{j}\right) \neq \eta_{m}(0) .
$$

We define $\lambda(\varepsilon)=\eta_{m}(0) \varepsilon^{-2}$. Since $\lambda_{j}(1) \rightarrow \infty$ as $j \rightarrow \infty$, we can find a number $i_{0}$ such that for any $i>i_{0}, \lambda_{i}(1)>\eta_{m}(0)$, and then $\lambda_{i}(1)-\lambda(1)=\lambda_{i}(1)-\eta_{m}(0)>0$. On the other hand,

$$
\lambda_{i}(\varepsilon)-\lambda(\varepsilon) \rightarrow-\infty \text { as } \varepsilon \rightarrow 0^{+}
$$

since $\lambda_{i}(\varepsilon)$ is a bounded function in $[0,1]$. Since $\lambda_{i}(\varepsilon)-\lambda(\varepsilon)$ is a continuous function, there exists a number $\varepsilon_{i}$ such that $\lambda_{i}\left(\varepsilon_{i}\right)=\lambda\left(\varepsilon_{i}\right)=\eta_{m}(0) \varepsilon_{i}^{-2}$. By (54), the sequence $\left\{\varepsilon_{i}\right\}_{i>i_{0}}$ cannot have subsequences converging to zero, so that $\inf _{i>i_{0}} \varepsilon_{i}=\varepsilon^{*}>0$. Take $\lambda^{*}=$ $\lambda\left(\varepsilon^{*}\right)=\eta_{m}(0)\left(\varepsilon^{*}\right)^{-2}$. The number of eigenvalues corresponding to $\varepsilon=\varepsilon^{*},\left\{\lambda_{i}\left(\varepsilon^{*}\right)\right\}_{i>i_{0}}$ 
smaller than $\lambda^{*}$, is finite, and we can find a number $i_{1}>i_{0}$ such that $\lambda_{i}\left(\varepsilon^{*}\right)>\lambda^{*}$ for each $i>i_{1}$. But, in this case,

$$
\lambda_{i}(\varepsilon)-\lambda(\varepsilon) \rightarrow-\infty \text { as } \varepsilon \rightarrow 0^{+},
$$

while

$$
\lambda_{i}\left(\varepsilon^{*}\right)-\lambda\left(\varepsilon^{*}\right)=\lambda_{i}\left(\varepsilon^{*}\right)-\lambda^{*}>0,
$$

and there must be some $\varepsilon_{i}<\varepsilon^{*}$ with $\lambda_{i}\left(\varepsilon_{i}\right)=\lambda\left(\varepsilon_{i}\right)$, which contradicts the definition of $\varepsilon^{*}$.

Lemma 10. For each integer $m \geq 1$, let $l^{m} \in \mathcal{L}$ be the family of Lemma 9 . The whole family $\left(\eta_{l_{\varepsilon}^{m}}\right)_{\varepsilon>0}$ converges to $\eta_{m}(0)$, as $\varepsilon \rightarrow 0$. In addition, if $\eta_{m}(0)$ is a simple eigenvalue of (23), then $\eta_{l_{\varepsilon}^{m}}(\varepsilon)$ is also a simple eigenvalue of (8) for $\varepsilon \leq \varepsilon_{0}$ small enough.

Proof. The proof is obtained using the theory of convergence of spectral families (cf. Sanchez-Hubert-Sanchez-Palencia [24]).

This finishes the proof of Theorem 2. Now, for more completeness, we give the strong interpretation of limit problem (23) in order to compare it to the classical spectral problems for torsion and stretching in rods (see (15) and (14)).

Proposition. For each integer $m \geq 1$, the limit solution $\left(\eta_{m}(0), \theta^{m}, \zeta^{m}\right)$ found out in Theorem 2 verifies:

i) If $\theta^{m} \neq 0$, then $\left(\eta_{m}(0), \theta^{m}\right)$ is an eigensolution of the classical torsional spectral problem

$$
-\mu J \theta^{\prime \prime}=\eta\left(I_{1}+I_{2}\right) \rho \theta \quad \text { in }(0, L), \quad \theta(0)=\theta(L)=0 .
$$

ii) If $\zeta^{m} \neq 0$, then $\left(\eta_{m}(0), \zeta^{m}\right)$ is an eigensolution of the classical stretching spectral problem

$$
-E \zeta^{\prime \prime}=\eta \rho \zeta \quad \text { in }(0, L), \quad \zeta(0)=\zeta(L)=0 .
$$

Proof. We obtain the result by performing an integration by parts in the right-hand side of (48).

6. Conclusion. We have proved that each torsional and stretching frequency of an elastic rod is the limit of a family of scaled frequencies of the three-dimensional elasticity model of the rod as the area tends to zero. Moreover, it was shown that this is the only limit that can be obtained with this scaling. We have also proved that the scaled three-dimensional eigenfunctions converge in a weak sense. If the limit is not zero, then it is a torsional or stretching eigenmode associated to the limit eigenvalue. However, the lack of strong convergence does not allow us to assure that the limit of eigenmodes is not zero. In this way, we provide a mathematical justification of classical equations for torsional and stretching vibrations. 
Acknowledgments. This work is part of the Human Capital and Mobility Program "Shells: Mathematical Modelling and Analysis, Scientific Computing" of the Commission of the European Communities (Contract No. ERBCHRXCT 940536) and of the Project: "Desarrollo de una teoría de hipervigas elásticas y su aplicación en ingeniería" of the Dirección General de Enseñanza Superior (DGES) of Spain (Ref. PB95-0862-C02-01). The authors are grateful to M. E. Pérez, E. Sanchez-Palencia, H. Le Dret, and E. Zuazua for many helpful discussions about content of this work.

\section{REFERENCES}

[1] I. Aganovic and Z. Tutek, A justification of the one-dimensional linear model of elastic beams, Math. Meth. Appl. Sci. 8, 1-14 (1986)

[2] J. A. Álvarez-Dios and J. M. Viaño, Mathematical justification of a one-dimensional model for general elastic shallow arches, Math. Meth. Appl. Sci. 21, 281-325 (1997)

[3] L. J. Álvarez-Vázquez and J. M. Viaño, Asymptotic justification of an evolution linear thermoelastic model for rods, Comput. Methods Appl. Mech. Engrg. 115, 93-109 (1994)

[4] A. Bermúdez and J. M. Viaño, Une justification des équations de la thermo-élasticité de poutres à section variable par des méthodes asymptotiques, RAIRO Anal. Numér. 18, 347-376 (1984)

[5] F. Bourquin and P. G. Ciarlet, Modelling and justification of eigenvalue problems for junctions between elastic structures, J. Funct. Anal. 87, 392-427 (1989)

[6] C. Castro and E. Zuazua, Une remarque sur l'analyse asymptotique spectrale en homogénéisation, C. R. Acad. Sci. Paris Sér. I Math. 322, 1043-1047 (1996)

[7] P. G. Ciarlet and S. Kesavan, Two-dimensional approximation of three-dimensional eigenvalue problems in plate theory, Comput. Methods Appl. Mech. Engrg. 26, 145-172 (1981)

[8] J. L. Davet, Correction du second ordre pour le calcul des fréquences propres d'une plaque en flexion, C. R. Acad. Sci. Paris Sér. II Math. 303, 521-524 (1986)

[9] V. Girault and P. A. Raviart, Finite Element Approximation of the Navier-Stokes equations, Lecture Notes in Mathematics, Vol. 749, Springer, Berlin, 1981

[10] I. Gruais, Modélisation de la jonction entre une plaque et une poutre en élasticité linéarisée, RAIRO Modél. Math. Anal. Numér. 27, 77-105 (1993)

[11] H. Irago, Comparación numérica de vibraciones $3 D-1 D$ en vigas elásticas, Tesina de Licenciatura de la Universidad de Santiago de Compostela, España, 1995

[12] H. Irago and J. M. Viaño, Second-order asymptotic approximation of flexural vibrations in elastic rods, Math. Models Methods Appl. Sci. 8, 1343-1362 (1998)

[13] N. Kerdid, Comportement asymptotique quand l'épaisseur tend vers zéro du problème de valeurs propres pour une poutre mince encastrée en élasticité linéaire, C. R. Acad. Sci. Paris Sér. I Math. 316, 755-758 (1993)

[14] N. Kerdid, Modélisation des vibrations d'une multi-structure formée de deux poutres, C. R. Acad. Sci. Paris Série I Math. 321, 1641-1646 (1995)

[15] N. Kerdid, Étude de problèmes de jonctions de poutres en élasticité linéaire, Thèse de Doctorat Université Pierre et Marie Curie, Paris, 1995

[16] N. Kerdid, Modeling the vibrations of a multi-rod structure, RAIRO Módel. Math. Anal. Numér. 31, 1-34 (1997)

[17] H. Le Dret, Modelling of the junction between two rods, J. Math. Pures Appl. 68, 365-397 (1989)

[18] H. Le Dret, Vibrations of a folded plate, RAIRO Módel. Math. Anal. Numér. 24, 501-521 (1990)

[19] H. Le Dret, Problèmes variationnels dans les multi-domaines. Modélisation des jonctions et applications, Recherches en Mathématiques Appliquées, Vol. 19, Masson, Paris, 1991

[20] V. Lods, Modélisation et justification d'un problème aux valeurs propres pour une plaque insérée dans un support tridimensionnel, C. R. Acad. Sci. Paris Sér. I Math. 320, 391-396 (1995)

[21] P. A. Raviart and J. M. Thomas, Introduction à l'Analyse Numérique des Équations aux Dérivées Partielles, Masson, Paris, 1983

[22] J. M. Rodríguez-Seijo and J. M. Viaño, Asymptotic derivation of a general linear model for thinwalled elastic rods, Comput. Methods Appl. Mech. Engrg. 147, 287-321 (1997)

[23] M. Roseau, Vibrations des systèmes méchaniques, Masson, Paris, 1984 
[24] J. Sanchez-Hubert and E. Sanchez-Palencia, Vibration and coupling of continuous systems. Asymptotic methods, Springer-Verlag, Berlin, 1989

[25] L. Trabucho and J. M. Viaño, Dérivation de modèles généralisés de poutres en élasticité par méthode asymptotique, C. R. Acad. Sci. Paris Sér. I Math. 304, 303-306 (1987)

[26] L. Trabucho and J. M. Viaño, Existence and characterization of higher-order terms in an asymptotic expansion method for linearized elastic beams, Asymptotic Analysis 2, 223-255 (1989)

[27] L. Trabucho and J. M. Viaño, A new approach of Timoshenko's beam theory by the asymptotic expansion method, RAIRO Módel. Math. Anal. Numér. 24, 651-680 (1990)

[28] L. Trabucho and J. M. Viaño, Mathematical modelling of rods, In Handbook of Numerical Analysis, Vol. IV, Ciarlet, P. G. and Lions, J. L., Editors, North-Holland, Amsterdam, 1996 